\title{
Limites des solutions solides dans le système binaire $\mathrm{CdBr}_{2}-\mathrm{AgBr}$
}

\author{
A. Wojakowska, A. Górniak and W. Gawel ${ }^{1}$ \\ Laboratoire d'Analyse Thermique, Département de Chimie Minérale, Académie de Médecine, \\ 38 rue Szewska, 50139 Wroclaw, Poland \\ ${ }^{1}$ Département de Chimie Analytique, Académie de Médecine, 38 rue Szewska, \\ 50139 Wroclaw, Poland
}

\begin{abstract}
RESUME. La limite inférieure du domaine d'existence de la solution solide $\mathrm{CdBr}_{2}$ dans $\mathrm{AgBr}$ s'étend de $\mathrm{AgBr}$ pur à $370 \mathrm{~K}$ jusqu'à la composition $40 \% \mathrm{~mol}$. $\mathrm{CdBr}_{2}$ à la température péritectique $715 \mathrm{~K}$. La solution solide $\mathrm{AgBr}$ dans $\mathrm{CdBr}_{2}$ s'étend entre les trois points: $\mathrm{CdBr}_{2}$ pur aux environs de $370 \mathrm{~K}$, la composition $4.5 \%$ mol. $\mathrm{AgBr}$ à l'invariance $715 \mathrm{~K}$ et le point de fusion de $\mathrm{CdBr}_{2}$. Les limites de ces domaines ont été déterminées par analyse thermique et conductivité électrique. La formation de la solution solide a été confirmée par les rayons $\mathrm{X}$.
\end{abstract}

\section{INTRODUCTION}

Quelques resultats sur le binaire $\mathrm{AgBr}-\mathrm{CdBr}_{2}$ ont été présentés pour la première fois par Zakharchenko [1]. Le diagramme de phase, fait à partir de douze échantillons, n'a montré que la courbe de liquidus, sans indiquer la composition du solide. Aucune solution solide n'a eté décelée.

Néanmoins, on peut rappeler qu'un bon nombre de données expérimentales relatives à la conductivité [2-4], diffusion [5,6], dilatation [7], force thermoélectrique [8,9] et chaleur specifique $[10,11]$ en fonction du dopage de $\mathrm{AgBr}$ par $\mathrm{Cd}^{2+}$ a eté présenté. Presque toutes ces études ont servi principalement à déterminer la nature et les propriétés des différents types de défauts du réseau cristallin. La teneur en dopant n'a pas excédé $1 \%$ mol. Cependant, les travaux de Vomhof et Nölting [10] ainsi que ceux de Teltow [2] ont démontré l'existence d'une solution solide plus étendue à base de $\mathrm{AgBr}$ à haute température, contenant pas moins de $8 \%$ mol. $\mathrm{CdBr}_{2}$ au-dessus de $500 \mathrm{~K}$ [10] et $30 \% \mathrm{~mol} \mathrm{CdBr}_{2}$ au dessus de $673 \mathrm{~K}[2]$.

Nous avons entrepris une étude plus systématique du système $\mathrm{AgBr}-\mathrm{CdBr}_{2}$ par $\mathrm{DSC}$, mesures de conductivité électrique et diffraction des rayons $\mathrm{X}$ afin d'élucider les équilibres entre phases dans ce système.

\section{PARTIE EXPERIMENTALE}

Le bromure d'argent a été préparé par la réaction de précipitation en solution aqueuse. Le bromure de cadmium a été déshydraté et distillé sous vide. Les mélanges de $\mathrm{AgBr}$ et $\mathrm{CdBr}_{2}$ ont été préparés en ampoules scellées sous vide.

L'analyse thermique est effectuée a l'aide d'un appareil Mettler Toledo DSC25 (logiciel STAR ${ }^{\mathrm{e}} 4.0$ ). Les mélanges des sels sont contenus dans des ampoules de silice, à fond plat, de $6 \mathrm{~mm}$ de diamètre et de $14 \mathrm{~mm}$ de hauteur, scellées sous vide. Les ampoules reçoivent environ $30 \mathrm{mg}$ de produit sauf pour certaines où le poids est dix fois plus grand. La vitesse de montée et de descente en température varie de $0.5 \mathrm{~K} \mathrm{~min}^{-1}$ à $20 \mathrm{~K} \mathrm{~min}^{-1}$.

Les mesures de conductivité sont réalisées généralement entre $400 \mathrm{~K}$ et $950 \mathrm{~K}$ à l'aide d'un condictomètre Taccusel CD 810 relié à un ordinateur. Les mélanges des sels, préalablement 
homogênéisés, sont contenus dans des cellules à capillaire à électrodes de platine [12]. La vitesse de montée et de descente en température ne depasse pas $1 \mathrm{~K} \mathrm{~min}^{-1}$.

Les diffractogrammes de rayons $X$ sont effectués à l'aide d'un appareil DRON-3 utilisant le rayonnement $\mathrm{K}_{\alpha}$ de l'anticathode de cuivre.

\section{RESULTATS}

Le diagramme des équilibres entre phases, que nous avons obtenu pour le système binaire $\mathrm{CdBr}_{2}-\mathrm{AgBr}$ est montré à la Fig. 1. Il est construit essentiellement à partir des courbes DSC. A quelques exceptions, les mesures de conductivité donnent les mêmes résultats.

Dans le système $\mathrm{CdBr}_{2}-\mathrm{AgBr}$ on retrouve un seul invariant à $715 \pm 2 \mathrm{~K}$ correspondant à la réaction péritectique:

$$
\mathrm{L}+\alpha=\beta
$$

où $\alpha$ - solution solide $\mathrm{AgBr}$ dans $\mathrm{CdBr}_{2}, \beta$ - solution solide $\mathrm{CdBr}_{2}$ dans $\mathrm{AgBr}, \mathrm{L}$ - liquide.

La limite inférieure du domaine d'existence de la solution solide $\mathrm{CdBr}_{2}$ dans $\mathrm{AgBr}$ (phase $\beta$ ) s'étend de $\mathrm{AgBr}$ pur aux environs de $370 \mathrm{~K}$ jusqu'à la composition $40 \% \mathrm{~mol} \mathrm{CdBr}_{2}$ à la température péritectique (Fig. 1). Cette frontière entre le domaine monophasé et biphasé se manifeste le plus clairement pendant une montée assez rapide en température (Fig. 2a) et pendant un refroidissement relativement lent (Fig. 2b). Ce changement de phase peut être également observé par conductimétrie (Fig $3 \mathrm{c}$ et $3 \mathrm{~d}$.).

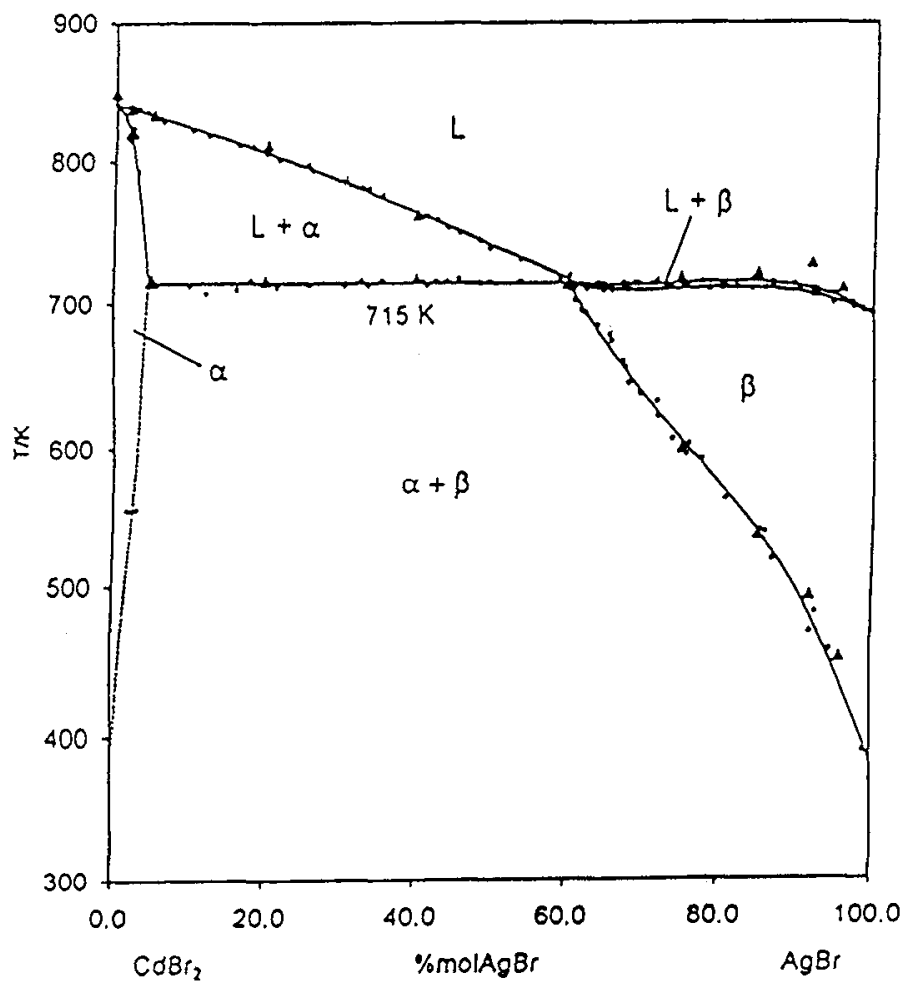

Fig. 1. Le diagramme d'équilibre entre phases pour le système $\mathrm{CdBr}_{2}-\mathrm{AgBr} ; \bullet \mathrm{DSC}, \mathbf{A}$ conductivité 

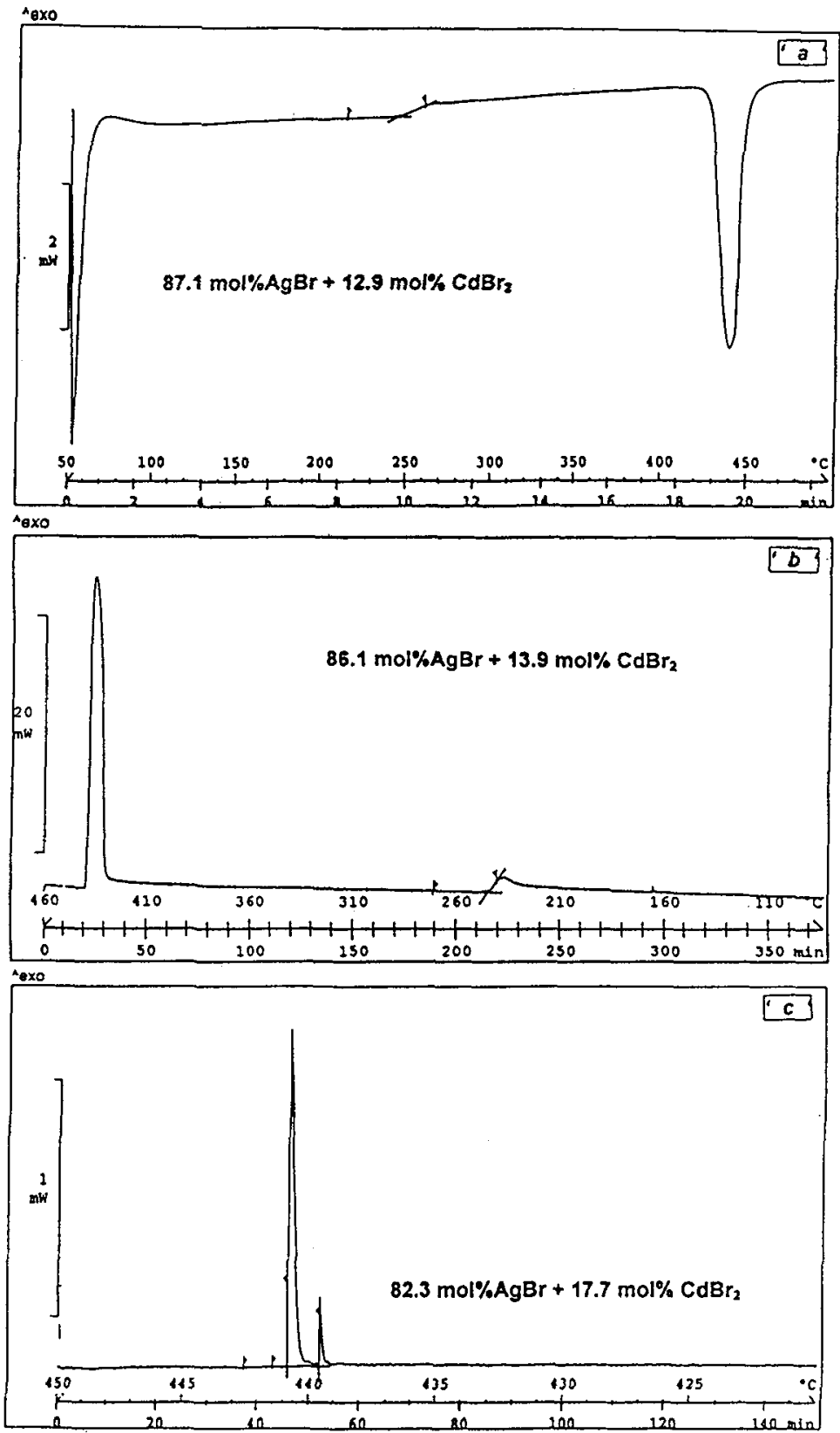

Fig 2. Exemples de courbes DSC: a). formation de la solution solide $\beta(87.1 \% \mathrm{~mol}$. AgBr) à $517.6 \mathrm{~K}$ en montée de température $20 \mathrm{~K} \mathrm{~min}^{-1}$; b). disparition de la solution solide $\beta(86.1 \% \mathrm{~mol}$. AgBr) à $519.2 \mathrm{~K}$ en descente de température $\left.1 \mathrm{~K} \mathrm{~min}^{-1} ; \mathrm{c}\right)$. separation des accidents thermiques $(82.3 \% \mathrm{~mol}$. AgBr $)$ relatifs au liquidus $(714.0 \mathrm{~K})$ et au solidus $(712.8 \mathrm{~K})$. 

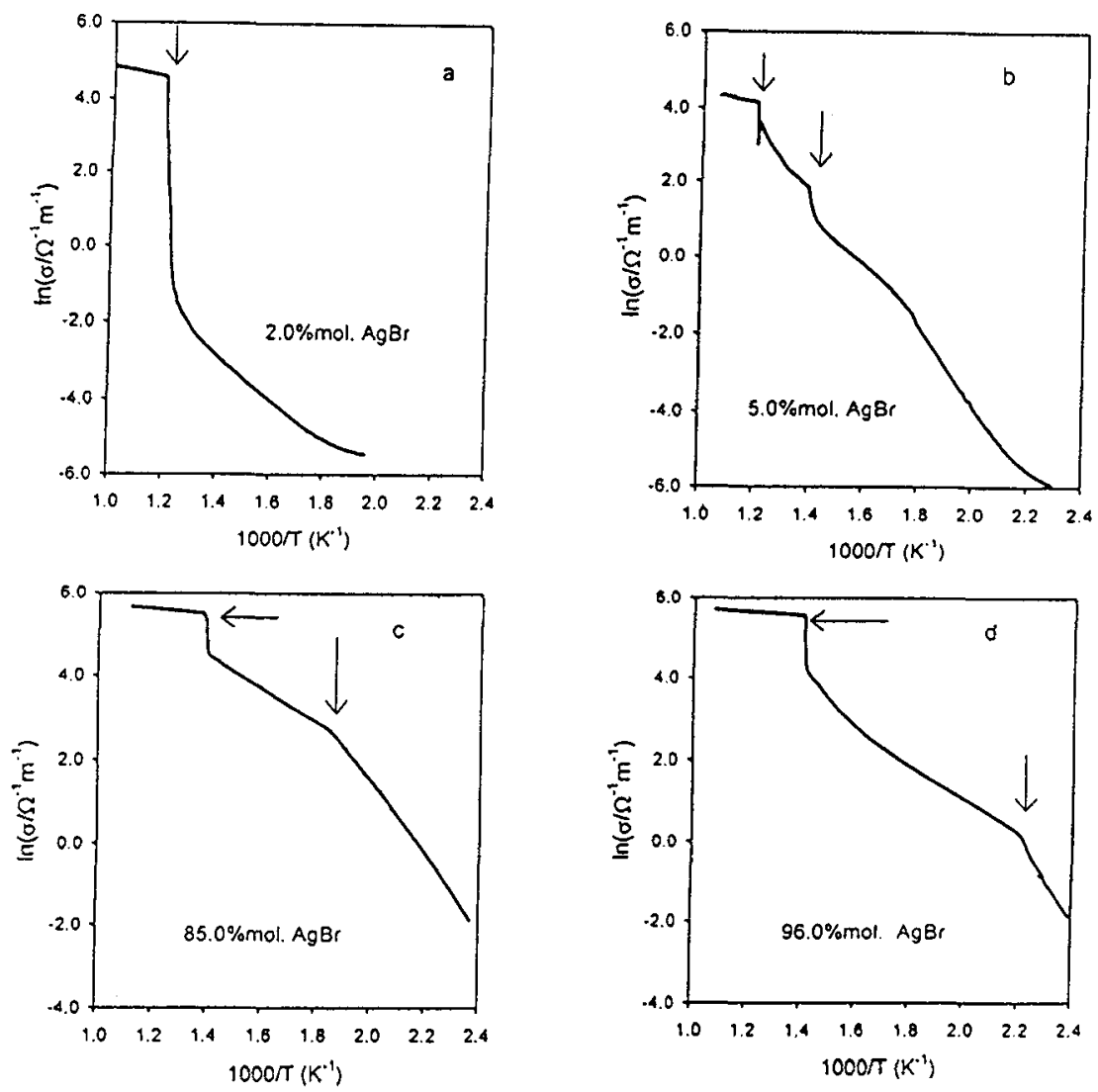

Fig. 3. Logarithme de la conductivité électrique en fonction de l'inverse de la température

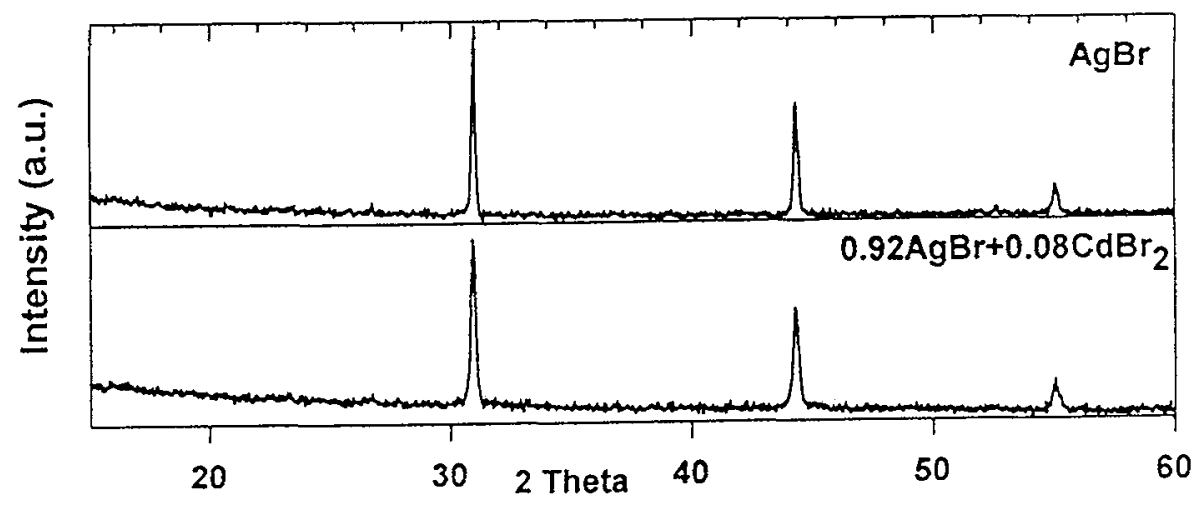

Fig 4. Diagrammes de diffraction $\mathrm{X}$ des échantillons ( $\mathrm{AgBr}$ pur et la solution solide contenant $92.0 \% \mathrm{~mol}$. AgBr) recuits à $650 \mathrm{~K}$ et trempés dans l'azote liquide. 
La courbe de solidus relative à la phase $\beta$ est très proche de celle de liquidus (Fig. 1). Deux accidents thermiques ont été observés seulement pour des échantillons refroidis à une vitesse de $1 \mathrm{~K} \mathrm{~min}^{-1}$ ou plus basse (Fig. 3c). La distance relative de deux accidents a été determinée avec une précision de $\pm 0.2 \mathrm{~K}$.

L'existence de la solution solide $\beta$ est démontrée aussi par diffraction des rayons X: le spectre obtenu pour un échantillon de $92,0 \% \mathrm{~mol} \mathrm{AgBr}$, recuit à $650 \mathrm{~K}$ pendant une semaine puis trempé dans l'azote liquide, ne contient que des raies de $\mathrm{AgBr}$, légèrement déplacées (Fig. 4).

La solution solide $\mathrm{AgBr}$ dans $\mathrm{CdBr}_{2}$ (phase $\alpha$ ) s'étend entre les trois points (Fig. 1.): $\mathrm{CdBr}_{2}$ pur aux environs de $370 \mathrm{~K}$, la composition $4.5 \% \mathrm{~mol} \mathrm{AgBr}$ à l'invariance $715 \mathrm{~K}$ et le point de fusion de $\mathrm{CdBr}_{2}$ $(841 \mathrm{~K})$. La solubilité maximale de $\mathrm{AgBr}$ dans $\mathrm{CdBr}_{2}$ a été évaluée à l'aide de mesures de conductivité. Sur les courbes DSC les accidents à $715 \mathrm{~K}$, caractéristiques de la péritexie, sont visibles jusqu'à la composition $10 \%$ mol $\mathrm{AgBr}$, puis disparaissent pour une teneur plus élevée en $\mathrm{CdBr}_{2}$, tandis que sur la courbe de conductivité (Fig. 3b) l'effet péritectique est observé encore pour la composition $5.0 \% \mathrm{~mol}$ AgBr. Il est absent pour les échantillons moins riches en bromure d'argent (Fig. 3a).

Une comparaison avec le binaire $\mathrm{CdCl}_{2}-\mathrm{AgCl}$ permet de noter des analogies étroites concernant des domaines d'existence des solutions solides à base des halogénures d'argent correspondants [13,14].

\section{References}

[1] Zakharchenko G. A., Zhurn. Obshch. Khim. 21 (1951) 453-456.

[2] Teltow J., Ann. Phys. (6) (Leipzig) 5 (1949) 63-70; $71-88$.

[3] Kurnick S. J., J. Chem. Phys. 20 (1952) 218-228.

[4] Hanlon J. E., J. Chem. Phys. 32 (1960) 1492-1500.

[5] Schöne E., Stasiw O., Teltow J., Z. Phys. Chem. 197 (1951) 145-160.

[6] Tannhauser D. S., J. Phys. Chem. Solids 5 (1958) 224-235.

[7] Zieten W., Z. Phys. 145 (1956) 125-130.

[8] Patrick L., Lawson A. W., J. Chem. Phys. 22 (1954) 1492-1495.

[9] Christy R. W., Fukushima E., Li H. T., J. Chem. Phys. 30 (1959) 136-138.

[10] Vomhof H. G., Nölting J., Ber. Bunsen-Ges. Phys. Chem. 79 (1975) 991-996.

[11] Lin P. L., Schmalzried H., Z. Phys. Chem. Neue Folge 99 (1976) 161-170.

[12] Wojakowska A., Kundys E., J. Mater. Sci. 25 (1990) 3780-3784.

[13] Blachnik R., Alberts J. E., Z. Anorg. Allg. Chem. 489 (1982) 161-172.

[14] Wojakowska A., Plinska S., Josiak J., Kundys E., High Temp. High Press. 30 (1998) 113-118. 